\title{
Nontuberculous Mycobacterial Infection Predisposing to Chronic Cavitary Pulmonary Aspergillosis
}

\author{
Bryan Vera Nieves ${ }^{1}$, Geoffrey Lindblad ${ }^{2}$, Benjamin Carmel $^{3}$, Andres Rivero ${ }^{4}$, Simon M. Edelstein ${ }^{4}$ \\ 1. Infectious Disease, Aventura Hospital and Medical Center, Aventura, USA 2. Radiology, Aventura Hospital and \\ Medical Center, Aventura, USA 3. Internal Medicine, Aventura Hospital and Medical Center, Aventura, USA 4. \\ Infectious Disease, Aventura Hospital and Medical Center, Miami, USA
}

Corresponding author: Bryan Vera Nieves, bryan.veranieves@hcahealthcare.com

\begin{abstract}
Aspergillus is a large group of spore-forming fungi in the phylum Ascomycota. Aspergillus infections more frequently occur in individuals with pre-existing lung conditions such as cystic fibrosis and asthma and immunosuppressed individuals, and less frequently in the immunocompetent population. Pulmonary aspergillosis can be subdivided into three categories: allergic bronchopulmonary aspergillosis, chronic pulmonary aspergillosis, and invasive pulmonary aspergillosis. We present a rare case of a 57 -year-old male with a previously known diagnosis of pancreatic adenocarcinoma on chemotherapy who was found to have a co-infection of the respiratory tract by Aspergillus flavus and Mycobacterium avium intracellulare.
\end{abstract}

Review began 06/28/2021 Review ended 07/08/2021 Published 07/16/2021

\section{Copyright 2021}

Vera Nieves et al. This is an open access article distributed under the terms of the Creative Commons Attribution License CC-BY 4.0., which permits unrestricted use, distribution, and reproduction in any medium, provided the original author and source are credited.
Categories: Internal Medicine, Radiology, Infectious Disease

Keywords: chest tube, aspergillosis, chronic cavitary pulmonary aspergillosis, atypical mycobacteria, mycobacterium avium intracellulare, pigtail catheter, mediport, nontuberculous mycobacteria

\section{Introduction}

Chronic pulmonary aspergillosis (CPA) comprises several different disease manifestations of chronic aspergillosis: simple aspergillomas, Aspergillus nodules, chronic cavitary pulmonary aspergillosis (CCPA), and chronic fibrosing pulmonary aspergillosis. CCPA, in particular, is characterized by one or more pulmonary cavities with or without aspergillomas, symptoms present for at least three months, and a positive Aspergillus immunoglobulin G (IgG) serology.

Mycobacterial infections are also capable of causing various pulmonary manifestations, particularly in immunosuppressed individuals. Both Aspergillus and Mycobacterium are opportunistic pathogens that can cause severe pulmonary disease, but co-infection of the respiratory tract with both pathogens is rarely reported in the literature [1]. It is estimated that up to 3 million people are affected by CPA worldwide [2]. Here, we present a rare case of a 57-year-old male with a previously known diagnosis of pancreatic adenocarcinoma on chemotherapy who was found to have co-infection of the respiratory tract by Aspergillus flavus and Mycobacterium avium intracellulare.

\section{Case Presentation}

A 57-year-old male with a past medical history pertinent for hyperlipidemia, pancreatic adenocarcinoma diagnosed six months prior and on chemotherapy, as well as recurrent bouts of pancreatitis presented to our institution due to shortness of breath, fever, chills, and epigastric abdominal pain radiating to his back and associated with nonbloody emesis that had been present for five days prior to arrival. Physical examination revealed a cachectic and frail man, with epigastric abdominal tenderness and voluntary guarding. Vital signs revealed a blood pressure of $102 / 63 \mathrm{mmHg}$, heart rate of 62 beats per minute, temperature of $36.9^{\circ} \mathrm{C}$, oxygen saturation of $99 \%$ at room air, and a respiratory rate of 16 breaths per minute. Initial laboratory studies revealed an elevated lipase of $1671 \mathrm{U} / \mathrm{L}(10-140 \mathrm{U} / \mathrm{L})$ and leukocytosis of $17.1 \times 10^{3} / \mathrm{uL}\left(4.0-10.5 \times 10^{3} / \mathrm{uL}\right)$ with a $75 \%$ neutrophilic predominance. A chest radiograph revealed a large right-sided apical cavitary lesion measuring up to $6 \mathrm{~cm}$ (Figure 1). 


\section{Cureus}

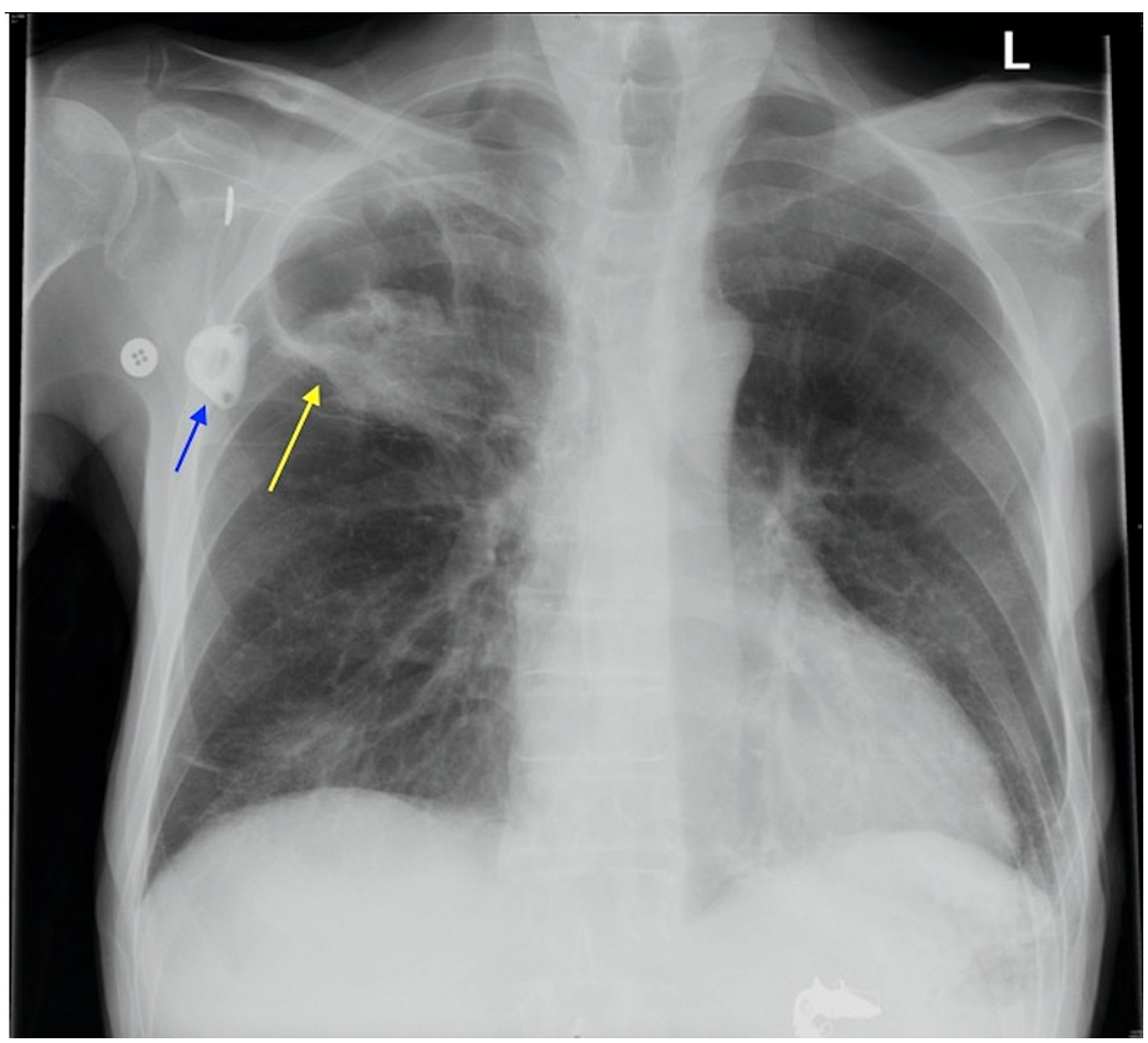

FIGURE 1: Chest radiograph demonstrating a right-sided apical cavitary lesion (yellow arrow) and a chemotherapy port (blue arrow).

A computed tomography (CT) scan of the chest with intravenous (IV) contrast was obtained to further characterize the findings evident on the chest radiograph which revealed a cavitary lesion with internal dependent debris and air-fluid level in the right upper lobe apical and anterior segment measuring $5.7 \times 5.7$ $\times 5.8 \mathrm{~cm}$ (Figure 2). 


\section{Cureus}

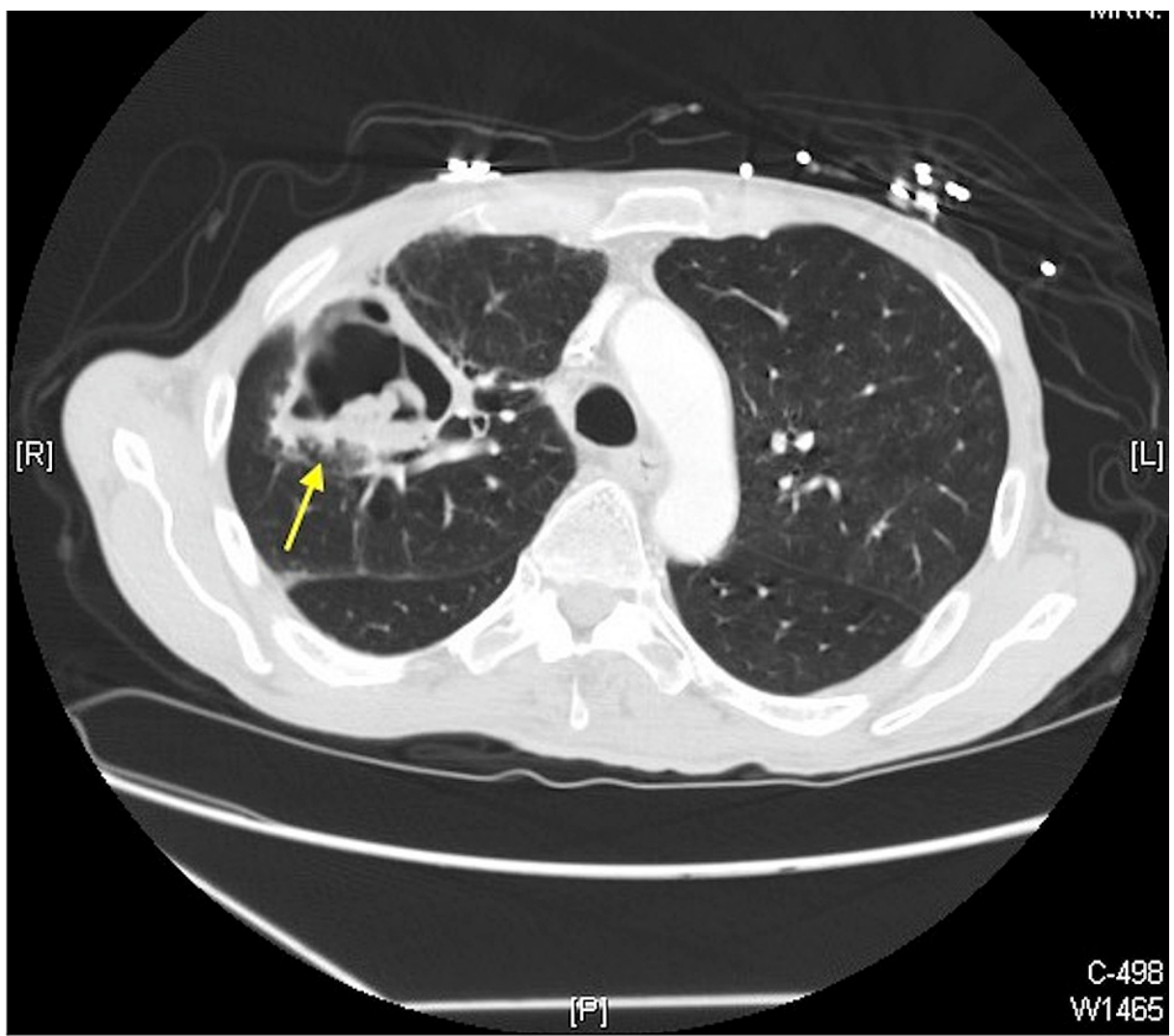

FIGURE 2: Axial reformatted CT chest with IV contrast demonstrating a cavitary lesion with internal dependent debris and an air-fluid level in the right upper lobe apical and anterior segment measuring $5.7 \times 5.7 \times$ $5.8 \mathrm{~cm}$ (yellow arrow).

CT: computed tomography; IV: intravenous

The patient was started on aggressive IV fluid hydration with Ringer's Lactate for the treatment of pancreatitis as well as IV piperacillin-tazobactam to cover for the possibility of bacterial cavitary pneumonia.

Bronchoscopy with bronchoalveolar lavage (BAL) was performed and fungal culture grew A. flavus with fungal smear revealing septate hyphae branching at 45-degree angles. A. flavus titers were elevated at 1:4, for which a diagnosis of CCPA was made. HIV testing was negative. Antifungal therapy with voriconazole $200 \mathrm{mg}$ PO BID was initiated. Piperacillin-tazobactam was discontinued as the BAL cultures were negative for bacteria. BAL acid-fast bacilli culture grew M. avium intracellulare and the patient was started on ethambutol $800 \mathrm{mg}$ PO QD, azithromycin $500 \mathrm{mg}$ PO BID, and amikacin $250 \mathrm{mg}$ IV BID.

The patient clinically improved with decreased oxygen requirement and down-trending white blood cell counts and was discharged from the hospital on voriconazole, ethambutol, azithromycin, and IV amikacin.

The patient returned to the emergency department at our institution with shortness of breath less than a week after being discharged. At this time, he had an oxygen saturation of $92 \%$ at room air, which improved to $97 \%$ with a $3 \mathrm{~L}$ nasal cannula. A chest radiograph showed evidence of a new right-sided spontaneous pneumothorax (Figure 3), which was also demonstrated on CT chest with IV contrast (Figure 4). 


\section{Cureus}

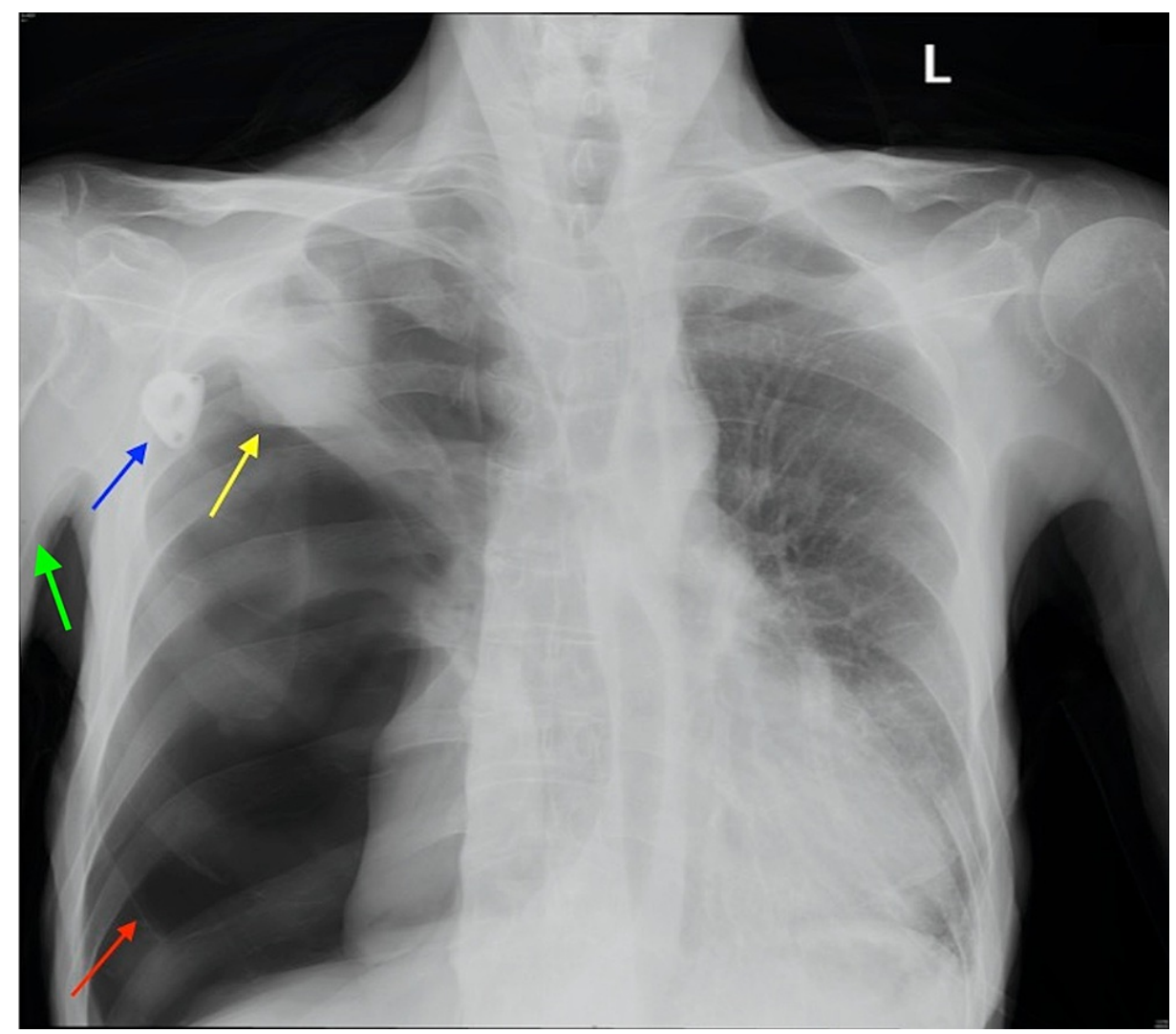

FIGURE 3: Chest radiograph demonstrating a right-sided pneumothorax (red arrow), a right-sided apical cavitary lesion (yellow arrow), a chemotherapy port (blue arrow), and a PICC line (green arrow). PICC: peripherally inserted central catheter

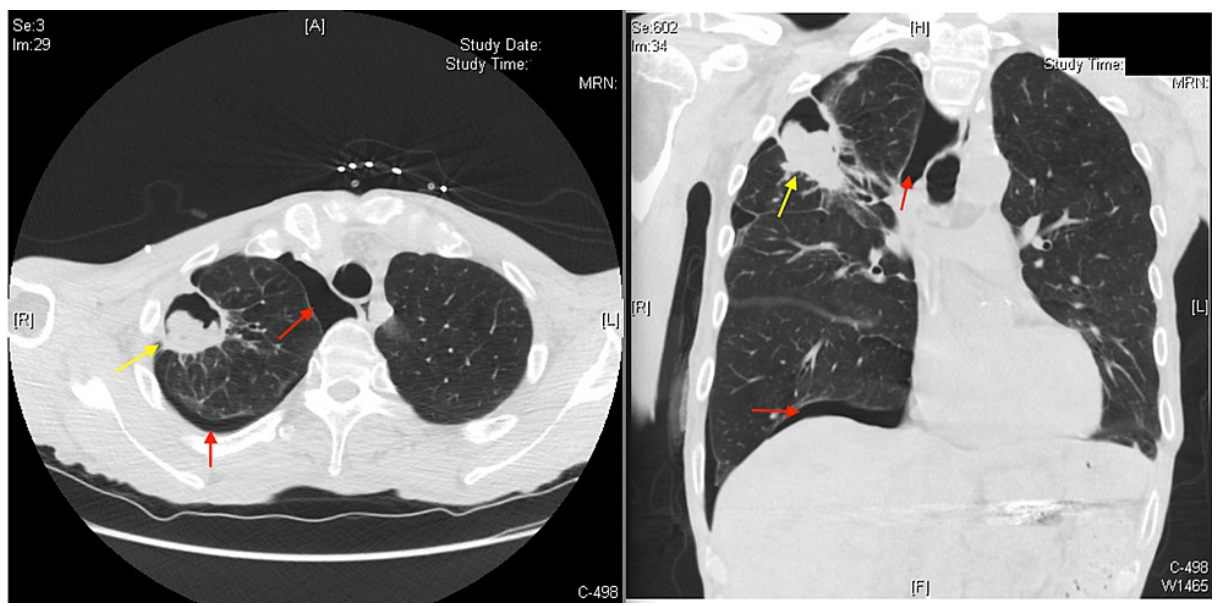

FIGURE 4: CT chest with IV contrast (axial reformatted: left image) (coronal reformatted: right image) demonstrating right-sided pneumothorax (red arrows) and right-sided apical cavitary lesion (yellow arrow).

CT: computed tomography; IV: intravenous

A right-sided chest tube was placed for the treatment of the right-sided pneumothorax (Figure 5). 


\section{Cureus}

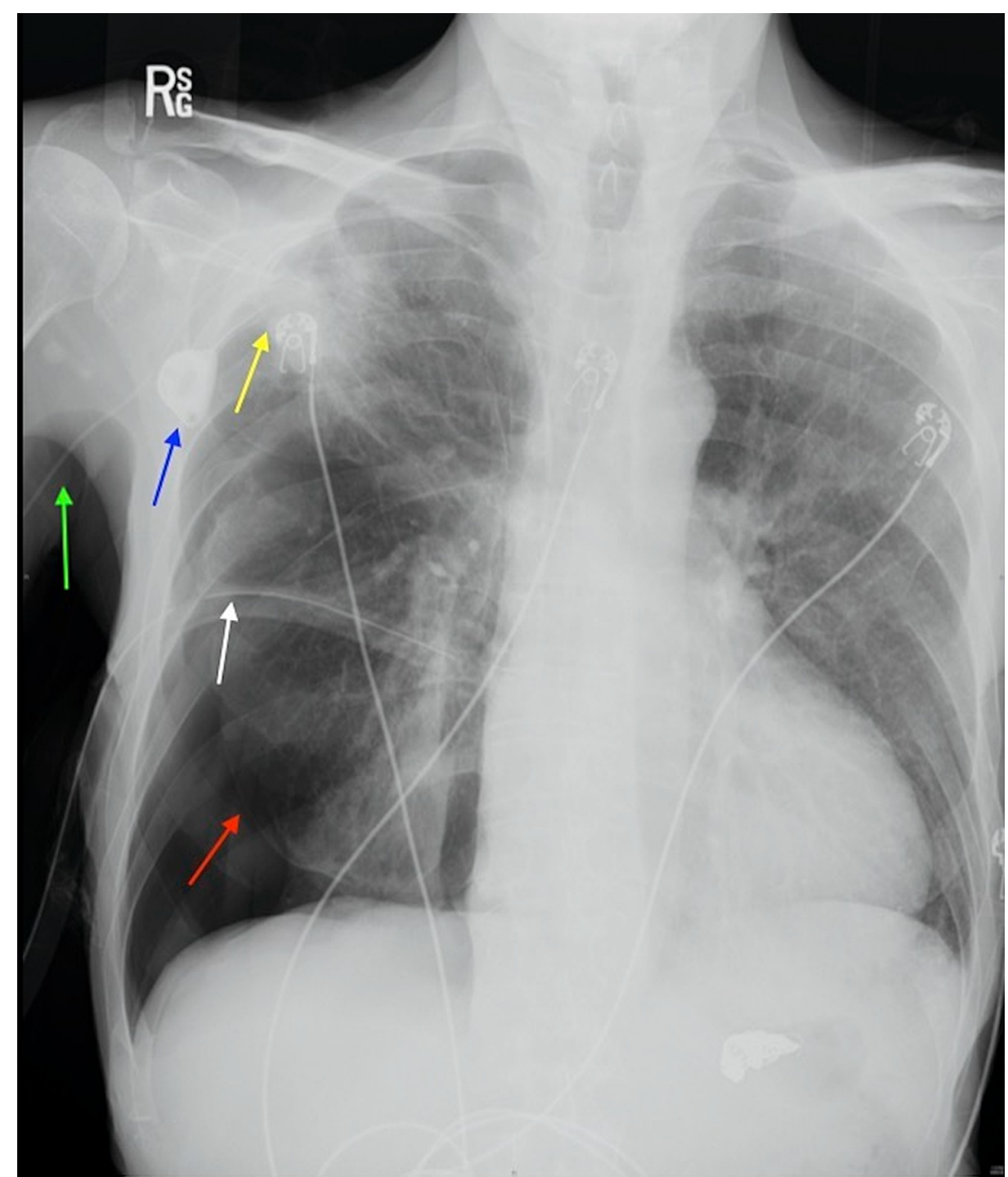

FIGURE 5: Chest radiograph demonstrating a right-sided pneumothorax (red arrow), a right-sided apical cavitary lesion (yellow arrow), a chemotherapy port (blue arrow), a PICC line (green arrow), and a chest tube (white arrow).

PICC: peripherally inserted central catheter

Daily chest radiographs were obtained to monitor the status of the pneumothorax. It continued to enlarge secondary to subsequent malpositioning of the chest tube. At this time, interventional radiology was consulted. A pigtail catheter was placed and the malpositioned chest tube was removed (Figure 6 ). 


\section{Cureus}

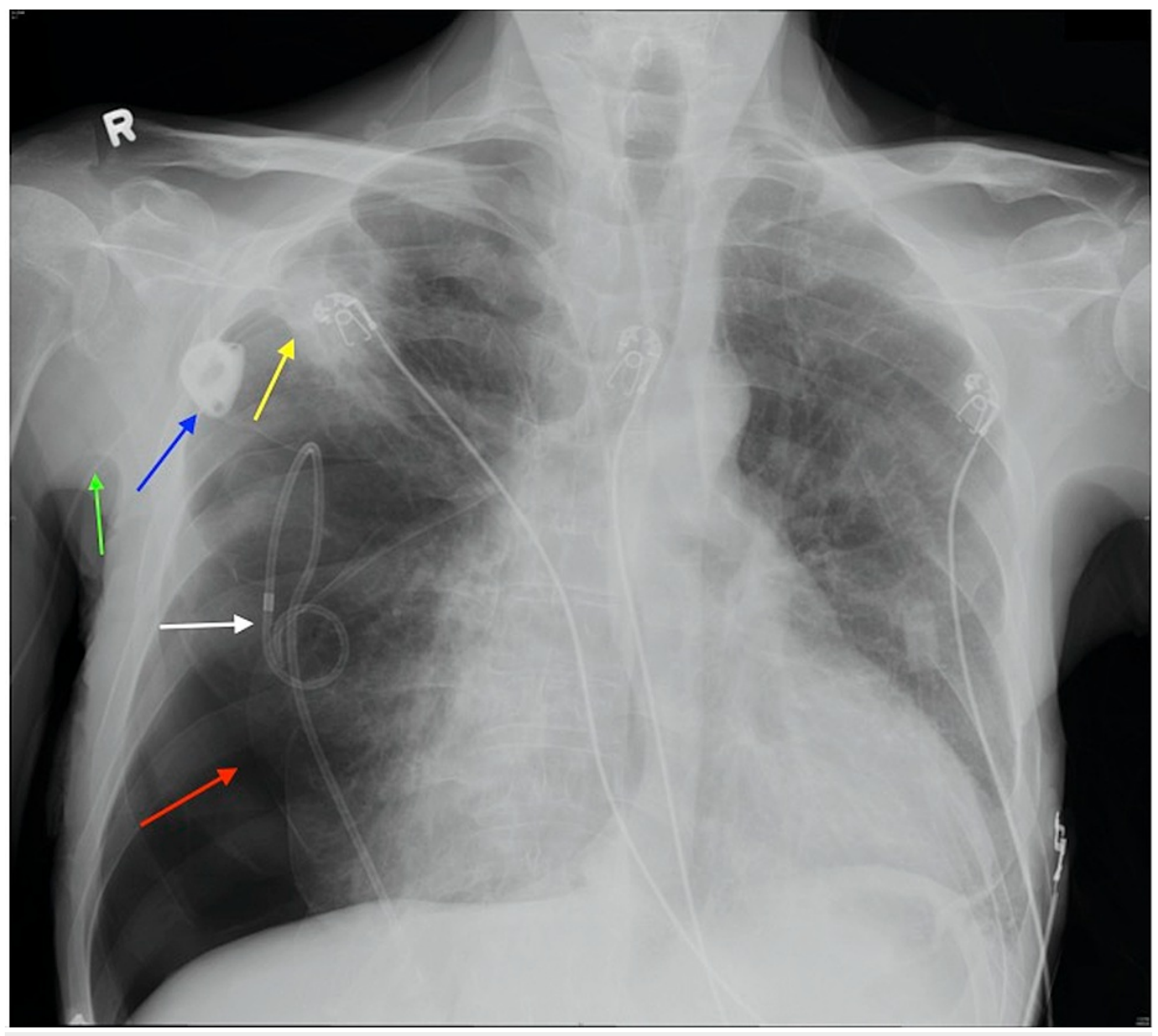

FIGURE 6: Chest radiograph demonstrating a right-sided pneumothorax (red arrow), a right-sided apical cavitary lesion (yellow arrow), a chemotherapy port (blue arrow), a PICC line (green arrow), and a pigtail catheter (white arrow).

PICC: peripherally inserted central catheter

Following pigtail catheter placement, the pneumothorax resolved within a week, and the catheter was removed without complications. A repeat chest radiograph demonstrated the resolution of the pneumothorax. The patient's oxygen saturation improved to $98 \%$ on room air.

Amikacin was changed from IV administration to the inhaled formulation to avoid nephrotoxicity on discharge. The patient was additionally sent home with voriconazole, azithromycin, and ethambutol. He was instructed to continue with close follow-up at our Infectious Disease Clinic.

\section{Discussion}

Co-infection in the respiratory tract between Aspergillus and Mycobacteria, particularly, nontuberculous mycobacteria (NTM) is a rare phenomenon, and its clinical significance has not been adequately defined and needs further research to help guide clinicians make clinical decisions when co-infection arises, as in our patient. Patients with NTM lung infection who also have CPA need to be identified by clinicians as they have worse clinical outcomes than those with NTM lung disease alone [3,4]. The majority of the patients with a mycobacterial infection who also have or later develop CPA typically have A. fumigatus or other Aspergillus species rather than A. flavus like in our patient [5-8]. Although A. flavus has not been traditionally mentioned as part of the diagnostic workup for CPA, our patient presenting with CPA secondary to A. flavus suggests there may be a benefit in including A. flavus serology in the diagnostic workup of CPA, even in areas where it is not typically endemic [9].

\section{Conclusions}

Although rare, CPA has been reported before in patients with NTM lung disease; recognizing this is extremely important for clinicians due to higher mortality in patients with NTM lung disease who are coinfected with CPA. A. flavus serology should be included in the diagnostic workup of patients with CPA due to its clinical significance, as demonstrated by our patient having CPA secondary to A. flavus. 


\section{Additional Information}

Disclosures

Human subjects: Consent was obtained or waived by all participants in this study. Conflicts of interest: In compliance with the ICMJE uniform disclosure form, all authors declare the following: Payment/services info: All authors have declared that no financial support was received from any organization for the submitted work. Financial relationships: All authors have declared that they have no financial relationships at present or within the previous three years with any organizations that might have an interest in the submitted work. Other relationships: All authors have declared that there are no other relationships or activities that could appear to have influenced the submitted work.

\section{Acknowledgements}

This research was supported (in whole or in part) by HCA Healthcare and/or an HCA Healthcare-affiliated entity. The views expressed in this publication represent those of the author(s) and do not necessarily represent the official views of HCA Healthcare or any of its affiliated entities.

\section{References}

1. Dellière S, Angebault C, Fihman V, et al.: Concomitant presence of Aspergillus species and Mycobacterium species in the respiratory tract of patients: underestimated co-occurrence?. Front Microbiol. 2019, 10:2980. 10.3389/fmicb.2019.02980

2. Barac A, Kosmidis C, Alastruey-Izquierdo A, Salzer HJ: Chronic pulmonary aspergillosis update: a year in review. Med Mycol. 2019, 57:S104-9. 10.1093/mmy/myy070

3. Naito M, Kurahara Y, Yoshida S, et al.: Prognosis of chronic pulmonary aspergillosis in patients with pulmonary non-tuberculous mycobacterial disease. Respir Investig. 2018, 56:326-31. 10.1016/j.resinv.2018.04.002

4. Takeda K, Imamura Y, Takazono T, et al.: The risk factors for developing of chronic pulmonary aspergillosis in nontuberculous mycobacteria patients and clinical characteristics and outcomes in chronic pulmonary aspergillosis patients coinfected with nontuberculous mycobacteria. Med Mycol. 2016, 54:120-7. 10.1093/mmy/myv093

5. Kousha M, Tadi R, Soubani AO: Pulmonary aspergillosis: a clinical review . Eur Respir Rev. 2011, 20:156-74. 10.1183/09059180.00001011

6. Stucky Hunter E, Richardson MD, Denning DW: Evaluation of LDBio Aspergillus ICT lateral flow assay for IgG and IgM antibody detection in chronic pulmonary aspergillosis. J Clin Microbiol. 2019, 57:e00538-19. 10.1128/JCM.00538-19

7. Sehgal IS, Choudhary H, Dhooria S, Aggarwal AN, Garg M, Chakrabarti A, Agarwal R: Diagnostic cut-off of Aspergillus fumigatus-specific IgG in the diagnosis of chronic pulmonary aspergillosis. Mycoses. 2018, 61:770-6. 10.1111/myc.12815

8. Hsu D, Irfan M, Jabeen K, et al.: Post tuberculosis treatment infectious complications. Int J Infect Dis. 2020, 92S:S41-5. 10.1016/j.ijid.2020.02.032

9. Jabeen K, Farooqi J, Iqbal N, Wahab K, Irfan M: Aspergillus fumigatus and Aspergillus flavus-specific IgG cut-offs for the diagnosis of chronic pulmonary aspergillosis in Pakistan. J Fungi (Basel). 2020, 6:249. 10.3390/jof6040249 